PROCEEDINGS OF THE

AMERICAN MATHEMATICAL SOCIETY

Volume 133, Number 6, Pages 1639-1646

S 0002-9939(05)07835-4

Article electronically published on January 25, 2005

\title{
INEQUALITIES FOR THE WEIGHTED MEAN OF $r$-CONVEX FUNCTIONS
}

\author{
MINGBAO SUN AND XIAOPING YANG \\ (Communicated by Carmen C. Chicone)
}

\begin{abstract}
In this paper, the inequalities for the weighted mean of $r$-convex functions are established. As applications, inequalities between the two-parameter mean of an $r$-convex function and extended mean values are given.
\end{abstract}

\section{INTRODUCTION}

The classical Hadamard's inequality for convex functions states that if $f:[a, b] \rightarrow$ $R$ is convex, then

$$
\frac{1}{b-a} \int_{a}^{b} f(t) d t \leq \frac{f(a)+f(b)}{2} .
$$

Following Hadamard, recently, the generalization of Hadamard's inequality to the integral power mean of a positive convex and $r$-convex function on $[a, b]$ are obtained by Pearce and Pečarić, and others (see [2, 5, 6]). The author extended Hadamard's inequality to the two-parameter mean of a positive convex function on $[a, b]$ (see 8]). The purpose of this paper is to extend Hadamard's inequality to the weighted mean of order $s$ of a positive $r$-convex function on $[a, b]$. As applications, inequalities between the two-parameter mean of a positive $r$-convex function and the extended mean values are given, which are more extensive results than the main results in $[2,5,6,6]$. Recall that a positive function $f$ is $r$-convex on an interval $I=[a, b]$ or $(a, b)$, if for all $x, y \in I$ and $\lambda \in[0,1]$,

$$
f(\lambda x+(1-\lambda) y) \leq \begin{cases}{\left[\lambda f^{r}(x)+(1-\lambda) f^{r}(y)\right]^{1 / r},} & \text { if } r \neq 0 \\ f^{\lambda}(x) f^{1-\lambda}(y), & \text { if } r=0\end{cases}
$$

The definition of $r$-convexity naturally complements the concept of $r$-concavity, in which the inequality is reversed (see [10]) and which plays an important role in statistics. We have that 0 -convex functions are simply log-convex functions and 1-convex functions are ordinary convex functions. For the latter the requirement that an $r$-convex function be positive clearly can be relaxed.

Received by the editors March 27, 2003.

2000 Mathematics Subject Classification. Primary 26D15, 26 A51.

Key words and phrases. Inequality, $r$-convex function, weighted mean, two-parameter mean, extended mean values, Tchebycheff integral inequality, standard mollifier.

This research was supported in part by SF for Pure Research of Natural Sciences of the Education Department of Hunan Province (no.2000C315), and NNSF (no.10271071) of China. 
Let $a, b, s \in R$, and $w$ and $f$ be positive and integrable functions on the closed interval $[a, b]$. The weighted mean of order $s$ of the function $f$ on $[a, b]$ with the weight $w$ is defined in [4] as

$$
M^{[s]}(f ; w ; a, b)=\left\{\begin{array}{cc}
{\left[\frac{\int_{a}^{b} w(t) f^{s}(t) d t}{\int_{a}^{b} w(t) d t}\right]^{1 / s},} & \text { if } s \neq 0, \\
\exp \left[\frac{\int_{a}^{b} w(t) \ln f(t) d t}{\int_{a}^{b} w(t) d t}\right], & \text { if } s=0 .
\end{array}\right.
$$

In addition, we define $M^{[s]}(f ; w ; a, a)=f(a)$. By taking $s=p-q, p, q \in R$, and replacing $w(t)$ by $w(t) f^{q}(t)$ in $(1.2)$, we get that $M^{[p-q]}\left(f ; w f^{q} ; a, b\right)=M_{w, f}(p, q ; a, b)$, where $M_{w, f}(p, q ; a, b)$ is the generalized weighted mean values of the function $f$ with weight $w$ and two parameters $p$ and $q$, which are defined in [7]. It is easy to see that the weighted mean $M^{[s]}(f ; w ; a, b)$ is equivalent to the generalized weighted mean values $M_{w, f}(p, q ; a, b)$. It is clear that the two-parameter mean $M_{p, q}(f ; a, b)$ of a positive function $f$ on $[a, b]$, which is given in [8], is a special case of $M_{w, f}(p, q ; a, b)$ applied to $w(t) \equiv 1$, while the integral power mean $M_{p}(f ; a, b)$ of a positive function $f$ on $[a, b]$ is a special case of $M_{p, q}(f ; a, b)$ applied to $q=0$. In particular, when $f(t)=t, x, y \in R^{+}$, the two-parameter mean $M_{p-1, q-1}(f ; x, y)$ is the extended mean value $E(p, q ; x, y)$ (see [9]) of two positive numbers $x, y$, which is given by $E(p, q ; x, x)=x$ if $x=y>0$ and for $x \neq y$ by

$$
\begin{gathered}
E(p, q ; x, y)=\left[\frac{q}{p} \frac{y^{p}-x^{p}}{y^{q}-x^{q}}\right]^{1 /(p-q)}, \quad p q(p-q) \neq 0, \\
E(p, 0 ; x, y)=E(0, p ; x, y)=\left[\frac{1}{p} \frac{y^{p}-x^{p}}{\ln y-\ln x}\right]^{1 / p}, \quad p \neq 0, \\
E(p, p ; x, y)=e^{-1 / p}\left(\frac{x^{x^{p}}}{y^{y^{p}}}\right)^{1 /\left(x^{p}-y^{p}\right)}, \quad p \neq 0, \\
E(0,0 ; x, y)=\sqrt{x y} .
\end{gathered}
$$

Clearly, $E(p+1,1 ; x, y)$ is the extended logarithmic mean $L_{p}(x, y)$ of two positive numbers $x, y$, while $E(p+1, p ; x, y)$ is also the alternative extended logarithmic mean $F_{p}(x, y)$ of two positive numbers $x, y$.

Our main results are the following four theorems.

Theorem 1.1. Let $f(t)$ be a positive and continuous function on an interval $[a, b]$ with continuous derivative $f^{\prime}(t)$ on $(a, b)$, let $g(x)$ be a positive and continuous function on the range $J$ of the function $f(t)$, and let $h(x) \equiv x$. Then if $f$ is r-convex,

$$
M^{[s]}(f ; g \circ f ; a, b) \leq M^{[s]}\left(h ; g h^{r-1} ; f(a), f(b)\right)
$$

for any real number $s$, while if $f$ is r-concave, the inequality is reversed.

Theorem 1.2. Let $f(t)$ be a positive and continuous function on an interval $[a, b]$, let $g(x)$ be a positive and continuous function on the range $J$ of the function $f(t)$, and let $h(x) \equiv x$. Then if $f$ is $r$-convex in the case of $r=0$ or 1 ,

$$
M^{[s]}(f ; g \circ f ; a, b) \leq M^{[s]}\left(h ; g h^{r-1} ; f(a), f(b)\right)
$$

for any real number $s$, while if $f$ is $r$-concave in the case of $r=1$, the inequality is reversed. 
As concrete applications, taking $g(x) \equiv x^{q}, x=f(t)$ on $[a, b]$, and $s=p-q, p, q \in$ $R$, in Theorems 1.1 and 1.2, respectively, we get the inequalities between the twoparameter mean $M_{p, q}(f ; a, b)$ of a positive $r$-convex function $f(t)$ on $[a, b]$ and the extended mean values $E(p+r, q+r ; f(a), f(b))$ of two positive numbers $f(a), f(b)$ as follows:

Theorem 1.3. Let $f(t)$ be a positive and continuous function on an interval $[a, b]$ with continuous derivative $f^{\prime}(t)$ on $(a, b)$. Then if $f$ is $r$-convex,

$$
M_{p, q}(f ; a, b) \leq E(p+r, q+r ; f(a), f(b))
$$

for any real numbers $p, q$, while if $f$ is $r$-concave, the inequality is reversed.

Theorem 1.4. Let $f(t)$ be a positive and continuous function on an interval $[a, b]$. Then if $f$ is $r$-convex in the case of $r=0$ or 1 ,

$$
M_{p, q}(f ; a, b) \leq E(p+r, q+r ; f(a), f(b))
$$

for any real numbers $p, q$, while if $f$ is $r$-concave in the case of $r=1$, the inequality is reversed.

Taking $q=0$ in Theorems 1.3 and 1.4, respectively, we can easily derive the following interesting corollaries:

Corollary 1.5. Under the hypotheses in Theorem 1.3 (or Theorem 1.4, respectively), if $f$ is r-convex, then

$$
\begin{array}{r}
M_{p}(f ; a, b) \leq E(p+r, r ; f(a), f(b)), \\
\frac{1}{b-a} \int_{a}^{b} f(t) d t \leq F_{r}(f(a), f(b))
\end{array}
$$

while if $f$ is $r$-concave, the inequalities are reversed.

Corollary 1.6. Suppose $f$ is a positive, continuous function on $[a, b]$. Then if $f$ is convex,

$$
M_{p}(f ; a, b) \leq L_{p}(f(a), f(b))
$$

while if $f$ is concave, the inequality is reversed.

Inequalities (1.7), (1.8), and (1.9) were established by Pearce and Pečarić, and others in [6, 2, 5], respectively. In 8], the author proved that inequality (1.5) is valid on the condition that $f$ is a positive and twice-differentiable 1-convex function. Therefore we obtain more extensive results than the main results in [2, 5, 6, 8].

Remark 1.7. If $s=1$ and $f$ is a twice-differentiable 1-convex function in Theorem 1.1 , it is easy to know that (1.3) is equivalent to

$$
M^{[1]}(f ; g \circ f ; a, b) \leq M^{[1]}(h ; g ; f(a), f(b))=\frac{\int_{a}^{b} g(f(t)) f^{\prime}(t) f(t) d t}{\int_{a}^{b} g(f(t)) f^{\prime}(t) d t} .
$$

In view of (1.10), we see that the corresponding conclusion of Theorem 1.1 may not hold provided the weight function $w$ in (1.2) does not satisfy the condition in Theorem 1.1. For example, putting $s=1, f(t)=t^{2}, w(t)=t+1, t \in[-1,3 / 5]$ in (1.2), we have that $f^{\prime}(t)=2 t, f^{\prime \prime}(t)=2>0$, but

$$
\frac{\int_{-1}^{3 / 5} w(t) f(t) d t}{\int_{-1}^{3 / 5} w(t) d t}>\frac{\int_{-1}^{3 / 5} w(t) f^{\prime}(t) f(t) d t}{\int_{-1}^{3 / 5} w(t) f^{\prime}(t) d t} .
$$


This paper, except for the introduction, is divided into two sections. In section 2 , we make some preparations. By using the result of section 2, we will give the proofs of Theorems 1.1 and 1.2 in section 3.

\section{SOME PRELIMINARY RESUlts}

In order to prove Theorems 1.1 and 1.2, we need the following Tchebycheff integral inequality (see [3, 4]).

Lemma 2.1. Let $p, q:[a, b] \rightarrow R$ be integrable functions, both increasing or both decreasing, and let $w:[a, b] \rightarrow R$ be a positive and integrable function. Then

$$
\int_{a}^{b} w(t) p(t) d t \int_{a}^{b} w(t) q(t) d t \leq \int_{a}^{b} w(t) d t \int_{a}^{b} w(t) p(t) q(t) d t .
$$

If one of the functions $p$ or $q$ is nonincreasing and the other nondecreasing, then the inequality in (2.1) is reversed.

We need some other notation: Let $U$ be an open subset of $R$. For $\epsilon>0$, we denote $U_{\epsilon} \equiv\{x \in U \mid \operatorname{dist}(x, \partial U)>\epsilon\}$. The function $\eta: R \rightarrow R$ is defined by

$$
\eta(x)= \begin{cases}c \exp \left[-1 /\left(1-|x|^{2}\right)\right], & \text { if }|x|<1 \\ 0, & \text { if }|x| \geq 1\end{cases}
$$

where $c$ is chosen so that $\int_{R} \eta(x) d x=1$. For $\epsilon>0$, define

$$
\eta_{\epsilon}(x) \equiv \frac{1}{\epsilon} \eta\left(\frac{x}{\epsilon}\right) \quad(x \in R)
$$

$\eta_{\epsilon}$ is the standard mollifier. If $f \in C(U)$, define

$$
f^{\epsilon} \equiv \eta_{\epsilon} * f
$$

that is,

$$
f^{\epsilon}(x) \equiv \int_{U} \eta_{\epsilon}(x-y) f(y) d y \quad\left(x \in U_{\epsilon}\right) .
$$

Lemma 2.2. If $f \in C(U)$, then for each $\epsilon>0, f^{\epsilon} \in C^{\infty}\left(U_{\epsilon}\right)$ and

$$
f^{\epsilon} \rightarrow f
$$

uniformly on compact subsets of $U$.

For the above notation and the proof of Lemma 2.2, the reader is referred to [1].

\section{Proofs of Theorem 1.1 and Theorem 1.2}

Proof of Theorem 1.1. Here we give only the proof in the case of $f(t)$ being $r$-convex for $r>0$ with continuous derivative $f^{\prime}(t)$ and $s>0$, since the proof in the other cases is similar.

(1). When $f(a) \neq f(b)$. Suppose that $G_{r}(x)$ is a primary function of $r x^{r-1} g(x)$ with respect to $x$, i.e. $G_{r}^{\prime}(x)=r x^{r-1} g(x)>0$ on the range of $f$. It is easy to know that $G_{r}(f(t))$ is also a primary function of $g(f(t))\left(f^{r}(t)\right)^{\prime}$. Then we have

$$
\int_{a}^{b} g(f(t))\left(f^{r}(t)\right)^{\prime} d t=G_{r}(f(b))-G_{r}(f(a)) \neq 0 .
$$

Similarly, we have that $\int_{a}^{b} g(f(t))\left(f^{r}(t)\right)^{\prime} f^{s}(t) d t \neq 0$ and

$$
\left(\int_{a}^{b} g(f(t))\left(f^{r}(t)\right)^{\prime} d t\right)\left(\int_{a}^{b} g(f(t))\left(f^{r}(t)\right)^{\prime} f^{s}(t) d t\right)>0 .
$$


It is easy to see that inequality (1.3) is equivalent to

$$
\frac{\int_{a}^{b} g(f(t)) f^{s}(t) d t}{\int_{a}^{b} g(f(t)) d t} \leq \frac{\int_{f(a)}^{f(b)} g(x) x^{r+s-1} d x}{\int_{f(a)}^{f(b)} g(x) x^{r-1} d x}=\frac{\int_{a}^{b} g(f(t))\left(f^{r}(t)\right)^{\prime} f^{s}(t) d t}{\int_{a}^{b} g(f(t))\left(f^{r}(t)\right)^{\prime} d t} .
$$

Let $\phi(t)=f^{r}(t)$, noticing that $f$ is $r$-convex for $r>0$ iff $f^{r}$ is convex, i.e., $\phi^{\prime}(t)=$ $\left(f^{r}(t)\right)^{\prime}$ is increasing. To prove the inequality (3.2), we consider the following three cases:

Case I. The derivative $\phi^{\prime}(t)=\left(f^{r}(t)\right)^{\prime}=r f^{r-1}(t) f^{\prime}(t) \geq 0$. Then $f^{\prime}(t) \geq$ 0 . Applying the Tchebycheff integral inequality (2.1) to the function $w(t)=$ $g(f(t)), p(t)=f^{s}(t)$ and $q(t)=\left(f^{r}(t)\right)^{\prime}$ for $t \in[a, b]$, we derive that inequality (3.2) holds.

Case II. The derivative $\phi^{\prime}(t)=\left(f^{r}(t)\right)^{\prime} \leq 0$. We obtain similarly that inequality (3.2) holds also by using the Tchebycheff integral inequality (2.1).

Case III. The sign of the derivative $\phi^{\prime}(t)=\left(f^{r}(t)\right)^{\prime}$ can be changed on $(a, b)$. If $f(a)<f(b)$, then $f^{r}(a)<f^{r}(b)$. Since $\phi^{\prime}(t)=\left(f^{r}(t)\right)^{\prime}$ is continuous and increasing, there exists a point $c \in(a, b)$ such that $\left(f^{r}(c)\right)^{\prime}=0,\left(f^{r}(t)\right)^{\prime} \leq 0$ for all $t \in(a, c)$ and $\left(f^{r}(t)\right)^{\prime} \geq 0$ for all $t \in(c, b)$. Therefore, there exists a point $d \in[c, b]$ such that $f^{r}(a)=f^{r}(d)$, i.e. $f(a)=f(d)$. Using (3.1), we have

$$
\int_{a}^{b} g(f(t))\left(f^{r}(t)\right)^{\prime} d t=\int_{d}^{b} g(f(t))\left(f^{r}(t)\right)^{\prime} d t
$$

similarly, we have

$$
\int_{a}^{b} g(f(t))\left(f^{r}(t)\right)^{\prime} f^{s}(t) d t=\int_{d}^{b} g(f(t))\left(f^{r}(t)\right)^{\prime} f^{s}(t) d t
$$

Thus the inequality (3.2) is equivalent to

$$
\frac{\int_{a}^{b} g(f(t)) f^{s}(t) d t}{\int_{a}^{b} g(f(t)) d t} \leq \frac{\int_{d}^{b} g(f(t))\left(f^{r}(t)\right)^{\prime} f^{s}(t) d t}{\int_{d}^{b} g(f(t))\left(f^{r}(t)\right)^{\prime} d t} .
$$

Denote $D=[a, b] \times[d, b], D_{1}=[a, d] \times[d, b], D_{2}=[d, b] \times[d, b]$. We consider

$$
\begin{aligned}
I= & \int_{a}^{b} g(f(x)) f^{s}(x) d x \int_{d}^{b} g(f(y))\left(f^{r}(y)\right)^{\prime} d y \\
& -\int_{a}^{b} g(f(x)) d x \int_{d}^{b} g(f(y))\left(f^{r}(y)\right)^{\prime} f^{s}(y) d y \\
= & \iint_{D} g(f(x)) g(f(y))\left(f^{r}(y)\right)^{\prime}\left[f^{s}(x)-f^{s}(y)\right] d x d y \\
= & S_{1}+S_{2},
\end{aligned}
$$

where

$$
S_{1}=\iint_{D_{1}} g(f(x)) g(f(y))\left(f^{r}(y)\right)^{\prime}\left[f^{s}(x)-f^{s}(y)\right] d x d y .
$$

When $(x, y) \in D_{1}$, we have $x \leq y$ and $\left(f^{r}(y)\right)^{\prime}=r f^{r-1}(y) f^{\prime}(y) \geq 0$ for all $y \in(d, b)$, i.e. $f^{\prime}(y) \geq 0$; thus $f^{s}(x) \leq f^{s}(d) \leq f^{s}(y)$. Therefore we have that $S_{1} \leq 0$. By the 
result proved in Case I, we can derive

$$
\begin{aligned}
S_{2}= & \iint_{D_{2}} g(f(x)) g(f(y))\left(f^{r}(y)\right)^{\prime}\left[f^{s}(x)-f^{s}(y)\right] d x d y \\
= & \int_{d}^{b} g(f(x)) f^{s}(x) d x \int_{d}^{b} g(f(y))\left(f^{r}(y)\right)^{\prime} d y \\
& -\int_{d}^{b} g(f(x)) d x \int_{d}^{b} g(f(y))\left(f^{r}(y)\right)^{\prime} f^{s}(y) d y \leq 0 ;
\end{aligned}
$$

therefore $I=S_{1}+S_{2} \leq 0$. It follows that (3.3) holds.

If $f(a)>f(b)$, by an argument similar to the proof in the case of $f(a)<f(b)$, we can get that (3.2) holds.

(2). When $f(a)=f(b)$, from the result proved in (1), we have that $\left(f^{r}(t)\right)^{\prime}$ is continuous and increasing, and there exists a point $c \in(a, b)$ such that $\left(f^{r}(c)\right)^{\prime}=$ $0,\left(f^{r}(t)\right)^{\prime} \leq 0$ for all $t \in(a, c)$ and $\left(f^{r}(t)\right)^{\prime} \geq 0$ for all $t \in(c, b)$. Hence $f^{s}(t) \leq f^{s}(b)$ for all $t \in(a, b)$. It follows that

$$
\int_{a}^{b} g(f(t)) f^{s}(t) d t \leq f^{s}(b) \int_{a}^{b} g(f(t)) d t .
$$

Therefore, the inequality (1.3) is valid.

This completes the proof of Theorem 1.1.

Proof of Theorem 1.2. We give only the proof in the case of $f$ being $r$-convex; if $f$ is $r$-concave, the proof is similar. We first consider the case when the origin $o$ belongs to $U=(a, b)$, i.e., $a<0<b$. For each $\epsilon>0$, write $U_{\epsilon}=(a+\epsilon, b-\epsilon)$, and define $f^{\epsilon} \equiv \eta_{\epsilon} * f$; that is, for $t \in(a+\epsilon, b-\epsilon)$,

$$
f^{\epsilon}(t)=\int_{U} \eta_{\epsilon}(t-y) f(y) d y,
$$

where $\eta_{\epsilon}$ is the standard mollifier. Since $f$ is continuous on $(a, b)$, from Lemma 2.2, we have that $f^{\epsilon}$ belongs to $C^{\infty}\left(U_{\epsilon}\right)$, and $f^{\epsilon} \rightarrow f$ uniformly on compact subsets of $(a, b)$. Now we prove for $\epsilon>0$ small enough that $f^{\epsilon}(t)$ satisfies the following conditions:

(i) For $t \in(a+\epsilon, b-\epsilon), f^{\epsilon}(t)=\int_{U} \eta_{\epsilon}(y) f(t-y) d y$.

(ii) $f^{\epsilon}(t) \in J$ for all $t \in(a+\epsilon, b-\epsilon)$.

(iii) $f^{\epsilon}$ is $r$-convex on $(a+\epsilon, b-\epsilon)$ in the case of $r=0$ or 1 .

In fact, for $t \in(a+\epsilon, b-\epsilon)$, we have

$$
\begin{aligned}
f^{\epsilon}(t) & =\int_{U} \eta_{\epsilon}(t-y) f(y) d y \\
& =\int_{B(t, \epsilon)} \eta_{\epsilon}(t-y) f(y) d y \\
& =\int_{B(0, \epsilon)} \eta_{\epsilon}(y) f(t-y) d y \\
& =\int_{U} \eta_{\epsilon}(y) f(t-y) d y,
\end{aligned}
$$

where $B(t, \epsilon)=\{y \in R|| t-y \mid<\epsilon\}$. This proves assertion (i). 
Let $M=\max \{f(t) \mid t \in[a, b]\}, m=\min \{f(t) \mid t \in[a, b]\}$. From the notation in section 2 , we have

$$
\begin{aligned}
f^{\epsilon}(t) & =\int_{U} \eta_{\epsilon}(t-y) f(y) d y \\
& \leq M \int_{B(t, \epsilon)} \eta_{\epsilon}(t-y) d y \\
& =M .
\end{aligned}
$$

Similarly, we can derive that $f^{\epsilon}(t) \geq m$ for $t \in(a+\epsilon, b-\epsilon)$. This completes the proof of assertion (ii).

Fix $x, y \in(a+\epsilon, b-\epsilon), \lambda \in[0,1]$. From the assertion (i), we derive

$$
\begin{aligned}
f^{\epsilon}(\lambda x+(1-\lambda) y) & =\int_{U} \eta_{\epsilon}(z) f((\lambda x+(1-\lambda) y)-z) d z \\
& =\int_{U} \eta_{\epsilon}(z) f(\lambda(x-z)+(1-\lambda)(y-z)) d z .
\end{aligned}
$$

In the case of $r=0$, from (3.4), (1.1) and Hölder's inequality, and applying the assertion (i), we obtain

$$
\begin{aligned}
f^{\epsilon}(\lambda x+(1-\lambda) y) & \leq \int_{U} \eta_{\epsilon}(z) f^{\lambda}(x-z) f^{1-\lambda}(y-z) d z \\
& \leq\left(\int_{U} \eta_{\epsilon}(z) f(x-z) d z\right)^{\lambda}\left(\int_{U} \eta_{\epsilon}(z) f(y-z) d z\right)^{1-\lambda} \\
& =\left(f^{\epsilon}(x)\right)^{\lambda}\left(f^{\epsilon}(y)\right)^{1-\lambda} .
\end{aligned}
$$

Similarly, from (3.4), (1.1) and the assertion (i), we derive that $f^{\epsilon}$ is also $r$-convex in the case of $r=1$.

This proves assertion (iii).

For any fixed $a^{\prime}, b^{\prime} \in(a+\epsilon, b-\epsilon)$ and $a^{\prime}<b^{\prime}$, and $\epsilon>0$ small enough, replacing $f$ by $f^{\epsilon}$ on $\left[a^{\prime}, b^{\prime}\right]$ in Theorem 1.1, we get

$$
M^{[s]}\left(f^{\epsilon} ; g \circ f^{\epsilon} ; a^{\prime}, b^{\prime}\right) \leq M^{[s]}\left(h ; g h^{r-1} ; f^{\epsilon}\left(a^{\prime}\right), f^{\epsilon}\left(b^{\prime}\right)\right) .
$$

Letting $\epsilon \rightarrow 0$, and $a^{\prime} \rightarrow a+, b^{\prime} \rightarrow b-$, we derive that (1.4) is valid in the case that the origin $o$ belongs to $(a, b)$.

We next consider the case in which the origin $o$ does not belong to $(a, b)$. For $t \in[a+e, b+e]$, we define

$$
F(t)=f(t-e),
$$

where $e$ is chosen so that the origin $o$ belongs to $(a+e, b+e)$. From the conditions of $f$ in Theorem 1.2, we can derive that $F(t)$ is a positive, $r$-convex and continuous function on $[a+e, b+e]$. Thus we obtain

$$
M^{[s]}(f ; g \circ f ; a, b)=M^{[s]}(F ; g \circ F ; a+e, b+e) .
$$

Replacing $f$ by $F$ on $[a+e, b+e]$ in inequality (1.4), proved in the case in which the origin $o$ belongs to $(a, b)$, we get

$$
M^{[s]}(F ; g \circ F ; a+e, b+e) \leq M^{[s]}\left(h ; g h^{r-1} ; F(a+e), F(b+e)\right) .
$$

From (3.6), (3.7), and noting $F(a+e)=f(a), F(b+e)=f(b)$, we derive that (1.4) is also valid in the case in which the origin $o$ does not belong to $(a, b)$.

This completes the proof of Theorem 1.2. 
Remark 3.1. From Theorems 1.1, 1.2, 1.3 and 1.4, it is natural to conjecture that (1.4) and (1.6) hold also for $r \neq 0,1$, respectively, under the hypotheses in Theorems 1.2 and 1.4 , respectively.

\section{ACKNOWLEDGEMENT}

We would like to thank the referee for valuable suggestions.

\section{REFERENCES}

[1] L. C. Evans and R. F. Gariepy, Measure Theory and Fine Properties of Functions, CRC Press, Boca Raton, 1992. MR.1158660 (93f:28001)

[2] P. M. Gill, C. E. M. Pearce, and J. Pečarić, Hadamard's inequality for r-convex functions, J. Math. Anal. Appl.215 (1997), 461-470. MR.1490762 (98k:26032)

[3] Ji-Chang Kung, Applied Inequallities, 2nd edition, Hunan Education Press, Changsha, China, 1993 (in Chinese). MF 95j:26001

[4] D. S. Mitrinović, Analytic Inequalities, Springer-Verlag, Berlin, 1970. MR0274686 (43:448)

[5] C. E. M. Pearce and J. Pečarić, A continuous analogue and an extension of Radó's formulae for convex and concave functions, Bull. Austral. Math. Soc. 53 (1996), 229-233. MR1381764 (97a:26023)

[6] C. E. M. Pearce, J. Pečaric and V. Šimić, Stolarsky means and Hadamard's inequality, J. Math. Anal. Appl. 220 (1998), 99-109. MR1612079 (99b:26040)

[7] Feng Qi, Generalized weighted mean values with two parameters, Proceedings of the Royal Society of London Series A-Mathematical, Physical and Engineering Sciences 454 (1998), no. 1978, 2723-2732. MR1650779 (99k:26027)

[8] Mingbao Sun, Inequalities for two-parameter mean of convex function, Math. Practice Theory 27 (1997), 193-197 (in Chinese). MR 1484124

[9] K. B. Stolarsky, Generalization of the logarithmic mean, Math. Mag. 48 (1975), 87-92. MR.0357718 (50:10186)

[10] B. Uhrin, Some remarks about the convolution of unimodal functions, Ann. Probab. 12 (1984), 640-645. MR0735860 (86b:60028)

Department of Applied Mathematics, Nanjing University of Science and Technology, Nanjing 210094, People's Republic of China - And - Department of Applied Mathematics, Hunan Institute of Science and Technology, Yueyang 414000, Hunan, People's Republic OF CHINA

E-mail address: sun_mingbao@163.com

Department of Applied Mathematics, Nanjing University of Science and Technology, NAnjing 210094, People's Republic of China 